\title{
Entomologisehe Nachriehten.
}

Begründet von Dr. F. Katter in Putbus.

Herausgegeben

von Dr. Ferd. Karsch in Berlin.

XIV. Jahrg.

Mai 1888.

Nr. 9.

\section{Cicindela Saxesenii Endrulat = farellensis Graëlls =} tartarica Mannerh. Bull. de Mosc. 1837. II. p. 10.

Gra ëlls unterscheidet ausdrücklich (Annal. France 1847. p. 311) eine var. maroccana Fabr. von der typischen campestris auct. und sagt alsdann von seiner farellensis: „supra rubro-cuprea; reliqua ut in typo". In der Abbildung ist seine farellensis besonders schmal. Daraus folgt unzweifelhaft, dass er eine purpurrothbraune campestris als farellensis beschrieben hat; meine farellensis zeigt nicht die dieser Varietät von G. Beuthin zugeschriebenen Eigenschaften; man denkt bei ihrem Anblick nicht an eine maroccana. Somit ist Herrn G. Beuthin's Ausspruch in diesen Blättern (No. 6 p. 81 ) nicht gerechtfertigt. Jedenfalls gehört aber farellensis Graëlls zu der 10 Jahre früher beschriebenen Cic. tartarica Mannerh., deren Hauptkennzeichen die Farbe „rubro-cuprea" ist; auch farellensis hat, ebenso wie Saxesenii, caput et thorax viridi-maculata. Ich habe den in alten Catalogen gebrauchten Namen Saxesenii Preller (in litt.) angewendet, um den unter diesem Namen bisher bezeichneten Käfer zu benennen. Der Endrulat'sche Name ist aber ohne Zweifel älter und berechtigt, da Endrulat die rothbraune Färbung, die den Hamburger Käfer kenntlich macht, ausdrücklich bei seiner Saxesenii erwähnt, deren Autor ihm unbekannt blieb.

Dr. G. Kraatz.

\section{Einige neue Ichneumoniden.}

Von Dr. Ferd. Rudow in Perleberg.

(Schluss).

H. Hinterleib schwarz mit rothen Mittelringen. (Gruppe Amblyteles Panzeri.)

a) Glied 2 roth.

25. A. tristis Rd.

Ater, opacus, alae nigrobrunneae, abdomen latum oviforme segmento secundo rufo. \& Long. corp. $22 \mathrm{~mm}$. 
Kräftig, gedrungen. Kopf breit, schwarz, Gesicht dicht grob punktirt. Fühler kurz, fast gleichmässig dick, einfarbig schwarz. Brustkasten dicht, grob runzelig punktirt, besonders der Hinterrücken runzelig. Schildchen vorn nadelrissig, hinten punktirt. Mittelfeld regelmässig quadratisch. Seiten des Hinterrückens stark höckerig mit kurzer Spitze versehen. Hinterleib kurz, breit eiförmig, Stiel stark nadelrissig, Luftlöcher auffallend klein und sehr weit entfernt von einander. Glied 2 ganz roth, die übrigen schwarz. Der Rücken der vorderen Glieder dicht punktirt, matt, die letzten feiner und etwas mehr glänzend. Bauchfalte dick, deutlich. Flügel ganz dunkelschwarzbraun, gleichmässig gefärbt. Beine kräftig, einfarbig schwarz. Hinterhüften unten mit dichter Haarbürste.

Aus Portugal stammend.

Ein $\$$ aus Südfrankreich stimmte mit dem erwähnten in allen Punkten überein, hat aber die hintere Hälfte des Schildchens gelbweiss gefärbt.

\section{A. lotharingicus Rd.}

Major, niger subnitidus, scutellum eburneum, pedes, coxis trochanteribus omnibus tarsisque posticis, secundum abdominis segmentum rufi, alae hyalinae venae brunneae, carpo rufo. \% Long. corp. $25 \mathrm{~mm}$.

Farbe schwarz, nur schwach glänzend. Kopf so breit wie der Brustkasten, schwarz, fein, dicht punktirt. Stirn stark ausgehöhlt. Fühler dünn, kurz, weiss geringelt und stark zugespitzt. Brustkasten seicht nadelrissig, Schildchen eben, gelblichweiss, Hinterrücken mit kräftigen, kurzen Seitendornen, stark runzelig. Mittelfeld fast quadratisch. Hinterleib wenig glänzend, Stiel längsrunzelig, am Ende punktirt. Luftlöcher rund, Ränder gekerbt, nicht sehr gross und weit von einander entfernt. Glied 2 ziegelroth, alle übrigen schwarz. Au Bauche Glied 2 und 3 roth, Falte undeutlich. Flügel wasserhell, Adern braunroth, Mal hellroth. Beine hell ziegelroth, alle Schenkelringe und Hüften, sowie die Hinterfüsse schwarz.

Stammt aus Lothringen und der Normandie.

\section{J. Hinterleib schwarz, Glied 2 und 3 roth, schwarz gefleckt.}

27. A. zonatus Rd.

Niger, subnitidus, alae flavescentes, carpo luteo, abdomen nigrum nitidum, segmento secundo et tertio rufis, margine postico nigris. 으. L. corp. $21 \mathrm{~mm}$. 
Schwarz, mässig glänzend. Kopf so breit wie der Brustkasten, Gesicht grob gerunzelt. Fühler schlank, dünn, stark zugespitzt, einfarbig schwarz. Brustkasten stark gerunzelt, Schildchen glatt eben, wenig punktirt, Hinterrücken sehr rauh, lang behaart. Mittelfeld fast quadratisch, Seitenränder nur sehr wenig gebogen. Hinterleib stärker glänzend, Stiel nadelrissig, Luftlöcher sehr klein. Glied 2 und 3 längsrunzelig, schön roth gefärbt mit schwarzen Hinterrändern. Ende schwarz, fein, dicht punktirt. Die beiden letzten Glieder bei einem Stück auf dem Rücken mit undeutlichen rothen Flecken. Bauch schwarz, glänzend, Falte deutlich. Flügel dunkelgelb, Adern braun, Mal gelbroth. Beine schlank, einfarbig schwarz, glänzend, Hinterhüften unten mit Haarbürste.

Aus der Normandie mit der Puppe eines Spinners erhalten.

\section{K. Hinterleib schwarz, Glied 2 und 3 ganz roth.}

28. A. mauritanicus $\mathrm{Rd}$.

Niger, nitidus, antennae alboannulatae, alae sat fumatae, pedes nigri, tibiis anticis subtus albis. Abdomen ovatum, segmentis 2 et 3 laete rufis. Long. corp. $21 \mathrm{~mm}$. 9 .

Kopf breit, Gesicht grob gerunzelt, steif behaart. Fühler kräftig, an der Spitze plötzlich zugespitzt mit schmalem, weissen Ring, der bei einem Stücke nicht ganz herum geht. Brustkasten oben fein gerunzelt, glänzend, Schildchen mit einzelnen Punkten, Brust und Hinterrücken stark gerunzelt, matt. Mittelfeld abgerundet, regelmässig. Flügel dunkelbraun, am Grunde fast schwarz. Hinterleib mässig glänzend, Stiel und Glied 1 grob nadelrissig, Luftlöcher sehr klein. Glied 2 dicht punktirt, die letzten fast glatt, stark glänzend. Farbe fast schwarz, Glied 2 und 3 von einem lebhaften Roth oben und unten. Der Hinterleib ist länger wie der Vorderleib und breit eiförmig. Bauchfalte nur vorn entwickelt.

Beine einfarbig schwarz, schlank, nur die Vorderschienen unten weiss gefärbt.

Aus Algier unter verschiedenen anderen Hymenopteren erhalten.

\section{A. severus $\mathrm{Rd}$.}

Niger, subnitidus, antennae setaceae unicolores, alae flavobrunneae, carpo luteo. Abdomen lateovatum, segmentis 2 et 3 laete rufis. Pedes nigri, crassiores, tibiae tarsique antici rufi. \& Long. corp. $24 \mathrm{~mm}$. 
Von Gestalt kräftig. Kopf breit, dicht borstig behaart. Gesicht fingerhutförmig punktirt. Fühler dünn, borstenförmig, einfarbig. Brustkasten dicht behaart, matt, sehr dicht grob punktirt, Schildchen eben, fast glatt. Hinterrücken grob gerunzelt, mit kräftigen, kurzen Seitendornen, Mittelfeld regelmässig quadratisch. Flügel einfarbig gelbbraun, Mal rothgelb. Hinterleib vorn mässig glänzend, Stiel grob nadelrissig, Luftlöcher gross mit starkgekerbten Rändern. Glied 2 und 3 dicht punktirt, lebhaft roth gefärbt, die letzten Glieder nur fein punktirt und stark glänzend. Bauchfalte deutlich entwickelt. Gestalt des Hinterleibes breit eiförmig, gedrungen. Beine lang mit dicken Schenkeln, fein, aber dicht borstig behaart, besonders die Hinterhüften. Vorderschienen und Fussglieder roth gefärbt.

Ein $\%$ hat einen schwarzen Längsstrich durch die 2 rothen Hinterleibsglieder und ist etwas kleiner mit fast elliptischem Hinterleibe.

Aus Südeuropa, Marseille und Palermo erhalten.

\section{A. massitiensis Rd.}

Niger, subnitidus dense pilosus. Antennae setiformes unicolores, thorax densissime punctatus, metathorace ruguloso bispinoso, scutello flavo, prothorace albomaculato. Alae subhyalinae, flavedine tinctae, carpo luteo. Abdomen elongatum, segmentis 2 et 3 rufis. Pedes nigri, pilosi, coxae albomaculatae, genua, tibiae tarsique antici rufi. Long. corp. $25 \mathrm{~mm}$. 90 .

Kopf mässig breit, dicht behaart, Gesicht buckelig erhaben, sehr dicht grob punktirt. Fühler dünn, lang, einfarbig. Brustkasten vorn stark gewölbt, dicht behaart und dicht grob punktirt, fast matt, nur die Brustseiten glänzend. Schildchen eben, dicht pnnktirt, gelblich. Ecken der Vorderbrust, Flügelschüppchen und Linien unter den Flügelwurzeln weiss. Hinterrücken grob gerunzelt mit kurzen, spitzen Seitendornen. Mittelfeld paralleltrapezisch, Seitenränder mässig gebogen. Hinterleib langgestreckt, fast doppelt so lang als der Brustkasten, dicht punktirt, matt, Glied 2 und 3 lebhaft roth. Bauchfalte deutlich. Flügel von bräunlichgelber Farbe, besonders an den Rändern, Mittelfläche heller, stark schillernd, Adern schwarz, Mal hellroth. Beine lang, alle Hüften mit weissgelbem Fleck und grob punktirt. Vorderknie, Schienbeine und Füsse röthlichgelb.

Das Männchen ist schlanker gebaut und hat eine Spur 
von rother Farbe am After, gleicht aber im übrigen dem Weibchen völlig.

Aus Marseille im Jahre 1885 erhalten.

L. Hinterleib schwarz mit rothem Ende. repentinus).

(Gruppe

31. A. bicolor Rd.

Ater, subnitidus, antennae, thorax, pedes posteriores, abdominis segmenta 1 et 2 nigra, pedes antici ex parte rufi, alae fumatae, carpo nigro. 0 \% Long. corp. $22 \mathrm{~mm}$.

\% Farbe schwarz, wenig glänzend. Gesicht querrunzelig, Kopf breit, dicht behaart. Fühler schwarz, borstenförmig, mit Spur von weissem Sattel. Brustkasten vorn stark buckelig gewölbt, dicht runzelig punktirt, nur die Brustseiten glänzend. Schildchen deutlich gewölbt, dicht grob punktirt mit weissem Fleck auf der Mitte. Hinterrücken sehr grob quergerunzelt mit starken, stumpfen Seitendornen. Mittelfeld quer rechteckig. Hinterleib mit Glied 1 und 2 schwarz, die anderen dunkelroth gefärbt. Stiel querrunzelig, Luftlöcher undeutlich klein. Glied 2 vorn längsrissig, hinten dicht punktirt, die übrigen fein punktirt, After glänzend. Beine schwarz, schlank, vordere Schenkel zur Hälfte, Schienbeine und Fussglieder roth. Flügel bräunlich, gleichmässig gefärbt, Mal und Adern schwarz.

ô hat ganz schwarze Fühler, schwarzes Schildchen und ausserdem an den mittleren Beinen die Schienen- und Fussglieder unten roth.

Stammt aus Italien, Sicilien und Griechenland.

M. Hinterleib roth, nur der Stiel schwarz. (Gruppe fuscipennis).

32. A. italicus Rd.

Inter majores, caput, thorax, femora, alae nigrae, antennae tricolores, tibiae tarsique omnes rufi, abdomen rufoflavum, stylo nigro. \& Long. corp. $27 \mathrm{~mm}$.

In der Gestalt grösser als die kräftigsten Stücke von fuscipennis, dem es auch sehr ähnlich ist. Kopf sehr breit, Gesicht querrunzelig und punktirt. Fühler kräftig, an der Spitze plötzlich verdünnt, dreifarbig. Sattel weiss, Spitze unten roth. Brustkasten mässig gewölbt, dicht grob punktirt. Schildchen stark gewölbt. Hinterrücken grob gerunzelt mit tief untenstehendem kurzem Seitenzahne. Mittelfeld schmal langgestreckt, vorn fast spitzwinkelig. Hinterleib hellmennigroth, Stiel schwarz, grob längsrunzelig, Luftlöcher länglich, 
gestreckt, einander genähert mit seicht gekerbten Seitenrändern. Alle Glieder matt, sehr dicht runzelig punktirt. Flügel dunkelschwarzbraun mit bläulichem Schiller, Mal dunkelbraun. Hinterhüften tiefgrubig punktirt. Alle Schenkel schwarz, alle Schienen roth, die Füsse unten röthlich gefärbt.

Das schöne Insekt habe ich vom verstorbenen Dr. Garbiglietti in $\mathrm{Tu}$ rin erhalten.

33. A. laticeps Rd.

Caput et thorax nigri nitidi, antennae setaceae alboannulatae, margines oculorum interni et externi et verticis, lineae ante scutellum flavae, abdomen et pedes purpurei, coxae et stylum nigrae. Alae subfumatae. \& Long. $19 \mathrm{~mm}$.

Kopf breiter als der Brustkasten, Stirne vertieft, fingerhutartig punktirt, Kiefer alle rothbraun gefärbt. Innere und äussere Augenränder schmal gelb gesäumt, Scheitel mit 2 gelben, kleinen Flecken. Fühler dünn, stark nach der Spitze verdünnt. Brustkasten oben sehr flach, dicht runzelig punktirt, Schildchen glänzend, eben, fein punktirt mit gelben Fleckchen vorn. Hinterrücken sehr grob gerunzelt, gewölbt, Mittelfeld klein quadratisch. Hinterleib purpurroth, mit dichter rauher Punktirung. Stiel schwarz, tief längsrissig, Luftlöcher länglich gross, Ränder glatt. Flügel schwach rauchgrau, Adern und Mal schwarz. Beine kräftig, Hinterschienen keulenförmig, mit Ausnahme der Hüften purpurroth gefärbt.

Vaterland It a lien.

Die Wespe hat Ähnlichkeit mit dem Kriechbaumer'schen $J$. platynotus aus Dalmatien, ist aber fast noch einmal so lang und viel kräftiger. $\mathrm{Ob}$ sie ein ächter Amblyteles ist, das muss die Vergleichung mit vielleicht gelegentlich $\mathrm{zu}$ erhaltenden Stücken lehren.

N. Hinterleib dreifarbig, Mittelring roth, After weissfleckig.

- 34. A. gynandromorphus Rd.

\% Niger subnitidus, antennae alboannulatae, margines oculorum interni rufi, scutellum flavum, metathorax bituberculatus. Abdominis segmenta 2 et 3 omnino, 4 ex parte rufa, 6 et 7 alboflavomaculata. Pedes nigri, tibiae tarsique antici subtus rufi. Alae maculatae flavescentes, carpo luteo. Long. corp. $21 \mathrm{~mm}$.

t facie flava, antennis nigris, abdominis segmentum tertium nigromaculatum, pedes nigri, tibiis anticis totis rufis, posticis rufoannulatis. Long. corp. $22 \mathrm{~mm}$. 
Q Kopf dick fast kugelig, Gesicht grob querrunzelig, innere Augenränder vom Scheitel an roth gesäumt. Fühler dick, an der Spitze plötzlich verdünnt, weiss geringelt. Brustkasten längsrunzelig punktirt, vorn stark buckelig, Schildchen mässig gewölbt, glänzend, gelb. Hinterrücken grob gerunzelt, stark gewölbt mit stumpfen Seitenhöckern, Mittelfeld fast quadratisch. Hinterleib breit, Stiel längsrissig, Luftlöcher länglich, Ränder glatt. Der Rücken ist dicht punktirt, matt, Glied 2 und 3 ganz ziegelroth, 4 an den vorderen Ecken, 6 und 7 weiss gefleckt. Bauchfalte deutlich entwickelt. Flügel gelblich, wolkig gefleckt, Mal rothgelb. Beine schwarz, Vorderschienen und Füsse unten röthlich.

đ Körpergestalt schlanker. Gesicht ganz gelb. Fühler schwarz mit gelbem Grundgliede vorn. Flügelschüppchen und Schildchen dunkelgelb. Hinterleib mit Glied 2 und 3 roth, letzteres mit 2 schwarzen Flecken am Vorderrande. Beine mit schwarzen Hüften und Schenkeln. Knie und Schienen nebst Füssen der zwei vorderen Paare rothgelb. Schienen der hinteren rothgelb mit schwarzen Spitzen. Füsse rothgelb, Klauenglied schwarz. Flügel gelblich mit grauem Rande.

Schmarotzer von Lasiocampa otus aus Südeuropa.

35. A. triguttatus Rd.

Niger nitidus, prothorax punctatissimus, linea sub alis scutelloque alboflavis, abdomen oviforme, segmento 2.3 rufis, 5. 6. 7 alboguttatis. Pedes nigri, tibiis anticis subtus alboflavis. Alae subhyalinae, margine fumato, carpo luteo. ㅇ Long. corp. $17 \mathrm{~mm}$.

Kopf fast kugelig, Stirn stark erhaben, grob punktirt. Fühler kurz, dünn, Spitze stark verdünnt. Brustkasten glänzend, sehr dicht fein punktirt, Linie unter dem Flügel weissgelb, ebenso das ebene Schildchen. Hinterrücken steil abfallend, grob punktirt, Mittelfeld halbmondförmig. Hinterleib kurz, breit eiförmig, Stiel fein nadelrissig, Luftlöcher klein. Der ganze Rücken stark glänzend. Glied 2 und 3 lebhaft roth, 5-7 mit weissem Rückenfleck. Bauchfalte deutlich. Flügel wasserhell, am Rande wenig getrübt, Mal gelbroth. Beine kräftig, schwarz. Vorderschienen und zum Theil -füsse unten rothgelb.

Aus der Normandie erhalten.

36. A. bizonatus Rd.

Niger subnitidus, caput cubicum, antennae tricolores, 
scutellum flavum. Abdomen subovale, segmento secundo rufo, tertii sextique margine postico late albo. Pedes rufi, coxis tibiisque posticis apice nigris. Alae flavescentes. ? Long. $17 \mathrm{~mm}$.

Der Kopf ist fast kugelig, das Gesicht erhaben, sehr grob punktirt. Fühler gleichmässig dick, dreifarbig. Brustkasten glänzend, dicht punktirt, Schildchen oben gelb, ebenso eine Linie unter den Flügeln und Flügelschüppchen. Hinterrücken steil abfallend, lang und dicht behaart. Hinterleib glänzend. Stiel nadelrissig, Luftlöcher sehr klein. Rücken dicht punktirt. Glied 2 ganz roth, 3 schwarz mit breitem weissen Hinterrande, 4 und 5 schwarz, 6 fast ganz weiss gefärbt. Flügel gleichmässig gelblich, mit gelbem Male. Beine rothgelb, Hüften und Spitzen der Hinterschienen schwarz.

Vaterland Normandie.

\title{
Ueber verschiedene hergebrachte Fehlernamen und Falsch- erklärungen in der Lepidopterologie.
}

\author{
Von Professor Dr. L. Glaser in Mannheim.
}

Der Einsender hat sich in diesen Blättern und besonders in seinem im selben Verlag erschienenen Catalogus etymologicus längere Zeit eingehend mit der Etymologie der hergebrachten Schmetterlingsnamen beschäftigt und ist bei seinen kritischen Untersuchungen mehrfach auf fehlerhafte Formen und so zu sagen stereotyp gewordene Fehlernamen gestossen. Indem er im Allgemeinen und Ganzen auf seinen erwähnten etymologischen Käfer- und Schmetterlingskatalog sich bezieht, will er im Interesse wissenschaftlicher Correctheit gewisse ständige, besonders änderungsbedürftige hervorhehen, die sich leider ,wie eine ewige Krankheit" fort und fort erhalten haben. Die Oertlichkeiten (nämlich Schriften, Kataloge und Preisverzeichnisse), worin sie vorkommen, jedesmal zu nennen, soll dabei unterlassen bleiben. Aber es ist wohl ein berechtigter Wunsch, dass offenbare Unrichtigkeiten künftig in solchen Oertlichkeiten vermieden und berichtigt werden, und Einsender will die fehlerhaften Namen nachstehend zur leichteren Orientirung alphabetisch vorführen. Es handelt sich namentlich um verwechselte Buchstaben (wie i und $\mathrm{y}, \mathrm{t}$ und th, $\mathrm{C}$ und $\mathrm{Ch}$ etc.), oder um grammatisch unrichtige Consonanten- $\mathrm{Zu}$ - 


\section{$2 \mathrm{BHL}$ Biodiversity Heritage Library}

Rudow, F. 1888. "Einige neue Ichneumoniden." Entomologische Nachrichten 14, 129-136. https://doi.org/10.5962/bhl.part.24114.

View This Item Online: $\underline{\text { https://www.biodiversitylibrary.org/item/42933 }}$

DOI: https://doi.org/10.5962/bhl.part.24114

Permalink: https://www.biodiversitylibrary.org/partpdf/24114

\section{Holding Institution}

Smithsonian Libraries

\section{Sponsored by}

Smithsonian

\section{Copyright \& Reuse}

Copyright Status: NOT_IN_COPYRIGHT

This document was created from content at the Biodiversity Heritage Library, the world's largest open access digital library for biodiversity literature and archives. Visit BHL at https://www.biodiversitylibrary.org. 